\title{
Non-invasive prenatal testing: the new era in reproductive medicine
}

\author{
Important advances in screening for genetic abnormalities have major \\ ethical implications
}

NIPT has been

embraced with

vigour by some

Australian

women and

health care

providers

Jan E Dickinson MD, FRANZCOG, DDU'

Caroline M de Costa PhD, MPH, FRANZCOG ${ }^{2}$

1 University of Western Australia, Perth, WA.

2 James Cook University School of Medicine, Cairns, QLD.

caroline.decosta@ jcu.edu.au

doi: 10.5694/mjal5.00299 n Australia, it is routine practice to offer pregnant women screening for fetal chromosomal and structural abnormalities, and, if serious anomalies are diagnosed, the option of terminating the pregnancy. Screening for chromosomal abnormalities commenced in the 1970s and was initially restricted to women whose pregnancies were considered to be of increased risk because of an obstetric history of aneuploidy or advanced maternal age. Over the past five decades, prenatal chromosome screening has been expanded to encompass the entire obstetric population. However, the commonly used aneuploidy screening tests are dogged by high false-positive rates, typically $4 \%-5 \%{ }^{1}$ Confirmation of an increased screening risk for aneuploidy involves diagnostic tests such as amniocentesis or chorionic villus sampling, each associated with low but definite risks of pregnancy loss.

Many women are reluctant to proceed with a diagnostic test after a positive aneuploidy screening result, ${ }^{2}$ and the ability to assess fetal genetic material without directly sampling the amniotic fluid or placenta has long been a goal of prenatal diagnosis. The recent development of non-invasive prenatal testing (NIPT), a high-level screening test using cell-free fetal DNA, offers the opportunity to markedly reduce the requirement for invasive testing while potentially also increasing detection rates of chromosomal anomalies, in particular of trisomy 21. In addition, NIPT may be offered earlier in pregnancy than standard aneuploidy screening and diagnostic techniques.

\section{Advances in DNA sequencing provide sensitivity and specificity}

The clinical introduction of NIPT for prenatal diagnosis was initially made possible by the recognition that cellfree fetal DNA (cffDNA) is detectable in the maternal bloodstream. cffDNA is placental in origin, released as the result of trophoblastic apoptosis, and is reliably detectable in maternal blood after about 10 weeks' gestation. ${ }^{3}$ Secondly, advances in DNA sequencing technology have led to the development of next-generation sequencing (NGS) techniques. DNA sequencing determines the exact order of nucleotides in a DNA fragment, and NGS enables millions of DNA fragments to be sequenced simultaneously. The use of massively parallel sequencing to precisely measure the relative abundance of sequences from each chromosome forms the basis of NIPT for aneuploidy screening. ${ }^{4,5}$ Further technical modifications have led to the introduction of more targeted approaches, the sequencing of selected regions of interest using single nucleotide polymorphism-based targeted assays, and,

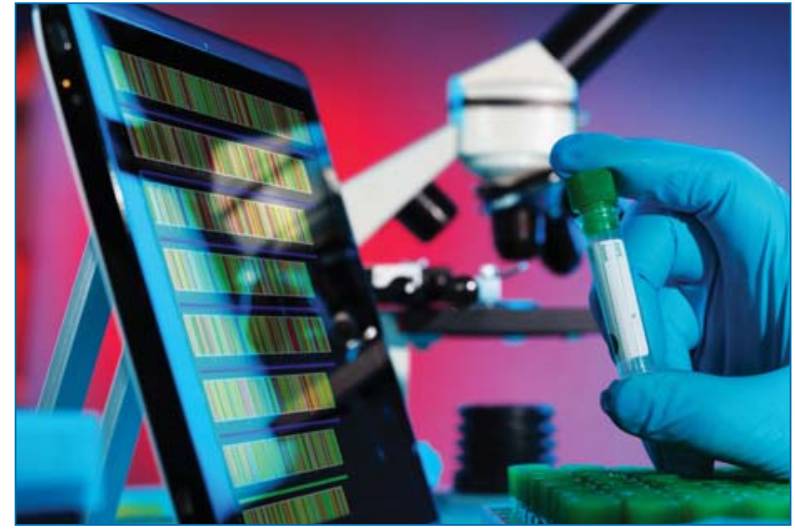

most recently, microarray-based cell-free DNA analysis. The focus of NIPT has progressively shifted from trisomy 21 to the detection of other trisomies and sex chromosomal aneuploidies. With the increase in the amount of sequencing data that can be obtained, the introduction of non-invasive testing for some chromosomal microdeletions and microduplications has become possible. These subchromosomal aberrations are much less common than the aneuploidies typically screened by existing tests, and these newer assays still require population studies to assess their performance characteristics. ${ }^{6}$

Although initially used in pregnancies at high risk for aneuploidy, recent data indicate that NIPT is also a robust screening test in lower-risk pregnancies. ${ }^{7} \mathrm{NIPT}$ is now the most sensitive and specific screening test for the common trisomies, with detection rates greater than $99 \%$ for trisomy 21 and false-positive rates of less than $0.5 \%$. The performance characteristics for trisomies 13 and 18 and the sex chromosome anomalies are lower than for trisomy 21, although the sensitivity is still typically greater than $90 \%{ }^{8}$

NIPT is currently available to Australian women from several international private companies on a user-pays basis, and a local provider has also recently offered the service. The price of testing varies between $\$ 420$ and $\$ 900$, and this is currently seen as too expensive for NIPT to be adopted as a publicly funded universal population screening strategy in Australia. Other, less expensive screening models have been proposed, and usually use maternal age and/or combined first trimester screening in a contingent screening model (typically using risk cut-offs of 1 in 1000-2000, capturing more than 95\% of fetal trisomy 21 cases). ${ }^{9,10}$

Since its initial introduction in late 2012, NIPT has been embraced with vigour by some Australian women and 
health care providers, ${ }^{11}$ it is available to any woman who is able to pay for it, regardless of medical risk level. ${ }^{12}$ There are strong arguments, both ethical and financial, for making NIPT available through the public health system in the near future, at least for major chromosomal anomalies. The inequity of access to NIPT and its current lack of regulation is a concern to many health care professionals.

\section{Caveats and limitations}

NIPT is not without its limitations. Failure to obtain a result occurs in routine clinical practice in about 3\%-4\% of tests, ${ }^{11}$ usually due to a low cffDNA fraction, typically because the sample was collected too early in the pregnancy or because of maternal obesity. False-positive results have been associated with confined placental mosaicism, the death of a co-twin, maternal malignancy and maternal mosaicism. Detection rates appear to be lower and the chances of not obtaining a result are higher in twin than in singleton pregnancies.

Ethical questions, ever present and never fully resolved when discussing prenatal testing, will come more sharply into focus in Australia, as elsewhere, with the broader introduction of NIPT into obstetric practice. A woman and her partner have two options after trisomy 21 has been diagnosed: continuation or termination of the pregnancy. The option of termination is widely regarded in our society as part of the couple's reproductive health rights. If the diagnosis is made earlier, and termination methods that are less stressful and safer for the woman and more acceptable to medical staff are available, there could be greater pressure to undergo testing (and termination, when abnormalities are detected) than is currently the case. What message does this then send to people with trisomy 21 in our community and their families? The same arguments would apply to other non-lethal chromosomal anomalies, such as Turner syndrome. Of even greater consequence would be the ability to discover the sex of the fetus at an early stage. Sex-based termination, widespread in some parts of Asia, is believed to be uncommon in Australia, but this situation could change were the sex known much earlier in pregnancy. The wider introduction of NIPT must be accompanied by appropriate increases in the provision of genetic counselling services for women and of education for health care providers.

The final goal of NIPT, although it may be some way off, will be sequencing of the entire fetal genome on a commercial basis, with the prospect of personalised prenatal diagnosis available to all, or at least to all who can afford it. Before this happens, it is important that we have widespread community understanding of all the questions involved, so that wise decisions can be made about how this testing should be made available to Australian women. ${ }^{12}$

\section{Competing interests: No relevant disclosures.}

Provenance: Commissioned; externally peer reviewed.

References are available online at www.mja.com.au.
1 O'Leary P, Breheny N, Dickinson JE, et al. First-trimester combined screening for Down syndrome and other fetal anomalies. Obstet Gynecol 2006; 107: 869-876.

2 Hadlow NC, Hewitt BG, Dickinson JE, et al. Community-based screening for Down's syndrome in the first trimester using ultrasound and maternal biochemistry. BJOG 2005; 112: 1561-1564.

3 Lo YM, Corbetta N, Chamberlain PF, et al. Presence of fetal DNA in maternal plasma and serum. Lancet 1997; 350: 485-487.

4 Chu RW, Chan KCA, Gao Y et al. Noninvasive prenatal diagnosis of fetal chromosomal aneuploidy by massively parallel genomic sequencing of DNA in maternal plasma. Proc Natl Acad Sci USA 2008; 105: 20458-20463.

5 Fan HC, Blumenfeld YJ, Chitkara U, et al. Noninvasive diagnosis of fetal aneuploidy by shotgun sequencing DNA from maternal blood. Proc Natl Acad Sci USA 2008; 105: 16266-16271.

6 Vora NL, O'Brien BM. Noninvasive prenatal testing for microdeletion syndrome and expanded trisomies: proceed with caution. Obstet Gynecol 2014; 123: 1097-1099.
7 Bianchi DW, Parker RL, Wentworth J, et al. DNA sequencing versus standard prenatal aneuploidy screening. N Engl J Med 2014; 370: 799-808.

8 Gil MM, Quezada MS, Revello R, et al. Analysis of cell-free DNA in maternal blood in screening for fetal aneuploidies: updated meta-analysis. Ultrasound Obstet Gynecol 2015; 45: 249-266.

9 O'Leary, P, Maxwell S, Murch A, Hendrie D. Prenatal screening for Down syndrome in Australia: costs and benefits of current and novel screening strategies. Aust N Z J Obstet Gynaecol. 2013: 53: 425-433.

10 Ayres AC, Whitty JA, Ellwood DA. A cost-effectiveness analysis comparing different strategies to implement noninvasive prenatal testing into a Down syndrome screening program. Aust N Z J Obstet Gynaecol 2014; 54: 412-417.

11 Hui L, Teoh M, da Silva Costa F, et al. Clinical implementation of cell-free DNA based aneuploidy screening: perspectives from a national audit. Ultrasound Obstet Gynecol. 2015; 45: 10-15.

12 Dickinson JE. Non-invasive prenatal testing: known knowns and known unknowns. Aust N Z J Obstet Gynaecol 2014; 54 : 397-399 = 